Henning Tesmer*, Roland Reese, Ersin Polat, Matthias Nickel, Rolf Jakoby, and Holger Maune

\title{
Fully Dielectric Rod Antenna Arrays with Integrated Power Divider
}

https://doi.org/10.1515/freq-2019-0152

Received March 13, 2019;

previously published online October 17, 2019

\begin{abstract}
This paper presents an overview of fully dielectric antenna arrays with integrated dielectric power dividers developed at Technische Universität Darmstadt as an extension of previous work. The power dividers are based on the principle of multimode interference and offer one- and two-dimensional power division in a single step, which allows the realization of small and lightweight devices. In order to prove the concept, $1 \times 4$ and $4 \times 4$ fully dielectric antenna arrays with integrated power dividers milled from Rexolite are designed, realized and characterized, operating between $90 \mathrm{GHz}$ and $105 \mathrm{GHz}$. To assess miniaturization and beamsteering capabilities, three $1 \times 4$ arrays composed of different materials, Rexolite, Preperm L300 and Preperm L440, are compared regarding gain, size, weight and element spacing. Depending on array size and material, gain is between $12.5 \mathrm{dBi}$ and $22 \mathrm{dBi}$, accompanied with sidelobe levels below -7.5 . All demonstrators are realized by milling, but the designs show the potential to be 3D printed or injection molded for large scale manufacturing.
\end{abstract}

Keywords: rod antenna, antenna array, millimeter wave devices, dielectric waveguide, multimode interference

\footnotetext{
*Corresponding author: Henning Tesmer, Institut für Mikrowellentechnik und Photonik, Technische Universität Darmstadt, Merckstraße 25, Darmstadt, 64289, Germany, E-mail: tesmer@imp.tu-darmstadt.de, https://orcid.org/0000-0002-5682-0812

Roland Reese, Institut für Mikrowellentechnik und Photonik, Technische Universität Darmstadt, Darmstadt, Germany, E-mail: reese@imp.tu-darmstadt.de

Ersin Polat: E-mail: polat@imp.tu-darmstadt.de, Matthias Nickel: E-mail: nickel@imp.tu-darmstadt.de, Institut für Mikrowellentechnik und Photonik, Technische Universität Darmstadt, Darmstadt, Hessen, Germany

Rolf Jakoby: E-mail: jakoby@imp.tu-darmstadt.de, Holger Maune: E-mail: maune@imp.tu-darmstadt.de, Institut für Mikrowellentechnik und Photonik, Technische Universität Darmstadt, Darmstadt, Germany
}

\section{Introduction}

The mm-wave regime is of special interest for high-data rate communication links and precise radar imaging, due to large effective bandwidths available at unassigned high frequency bands. Devices operating in the upper $\mathrm{mm}$ wave region are therefore paving the way for future communication systems and are closing the gap between the Gigahertz and Terahertz spectrum. However, the increasing path loss is the main obstacle to overcome in order to be able to exploit the afore mentioned benefits of a high operating frequency. In order to compensate path loss, high-gain antenna arrays can be used. In addition, antenna arrays offer the possibility to perform beam steering by creating a phased array. Therefore, the potentially narrow beam of the array can be steered towards a desired direction, establishing a stable data link even for nonstationary scenarios.

At mm-wave frequencies, the ohmic losses of metallic waveguides and antennas become a major issue when designing waveguiding devices. These losses can be avoided by using dielectric waveguides (DWGs). DWGs offer a lightweight, low loss, cost efficient and easy to fabricate alternative to conventional waveguides. They have been widely utilized at millimeter waves in various devices such as flexible transmission lines [1], [2] or tunable phase shifters [3-6]. A simple way to realize a dielectric antenna is to taper the DWG at its end, such that a dielectric rod antenna (DRA) is formed [7]. Due to their design, DRAs are broadband, easy to match, and fabrication can be kept simple and cost efficient. Furthermore, gain and beamwidth can be tuned by changing the DRA's taper length solely, hence, no fundamental change in the different antennas' shapes is necessary for customization [7]. Together with dielectric phase shifters, e.g. [3],[4], the tunable antenna can be realized in a fully dielectric manner.

In order to feed the different dielectric antenna elements a power distribution network is needed. A hollow waveguide feed such as in [8], [9] could be an option, but it is comparably heavy and has to be assembled with individual metallic and dielectric parts. A combination of a metallic and a dielectric approach has been shown in [10], where the dielectric is electroplated. However, the 
advantage of solely dielectric losses is no longer given. Classical dielectric Y-dividers [11, 12] offer the possibility to split power fully dielectric both in $x$ - and $y$-direction, however, they are fragile and consume a lot of space since the bending radii of the fibers have to be very smooth in order to avoid parasitic radiation at discontinuities and bends.

A compact and elegant way to circumvent the aforementioned drawbacks is to make use of multimode interference (MMI) [13], [14]. Originally utilized in the optical domain, its principle can be adapted to the mm-wave region, allowing compact integrated power splits in one solid, mechanically robust block of dielectric material. The basic concept of MMI is the fact that a sudden broadening of the DWG leads to the propagation of higher-order modes along the broad section of the DWG. Since these modes have different propagation constants, a varying interference pattern is formed along the multimode waveguide. At distinct lengths, separated maxima of evenly distributed power arise. Placing single-mode output DWGs at the positions of these maxima allows splitting the power evenly between each output DWG.

In this manuscript the basic concept of MMI is reviewed, before its application as a power divider for antenna arrays is discussed, based on [15, 16] and [17]. Both one- and two-dimensional antenna arrays are simulated, designed, realized and measured by using a low-loss dielectric with easy processing capabilities and a permittivity of $\varepsilon_{r}=2.53$. In the two-dimensional case, a flexible measurement setup is introduced in order to verify equal power split, showing how the different output ports behave regarding power imbalance and phase distribution. To assess its miniaturization and beamsteering capabilities, a feasibility analysis is carried out with three realized one-dimensional arrays made from three different materials with permittivities of $\varepsilon_{r}=2.53, \varepsilon_{r}=3.0$ and $\varepsilon_{r}=4.4$. The results together with the possible extension to fully dielectric phased arrays with integrated power divider are summarized at the end of this paper.

\section{Multimode interference}

Multimode interference (MMI), well described in [13], was initially used in the optical domain for couplers and dividers [14]. It is often referred to as self-imaging, since the input field distribution is repeated along a multimode waveguide. Figure 1 shows exemplary the first 5 modes of a rectangular dielectric waveguide of width $w=12.25 \mathrm{~mm}$, height $h=1.8 \mathrm{~mm}$ and $\varepsilon_{r}=2.53$, operating in W-Band. Due

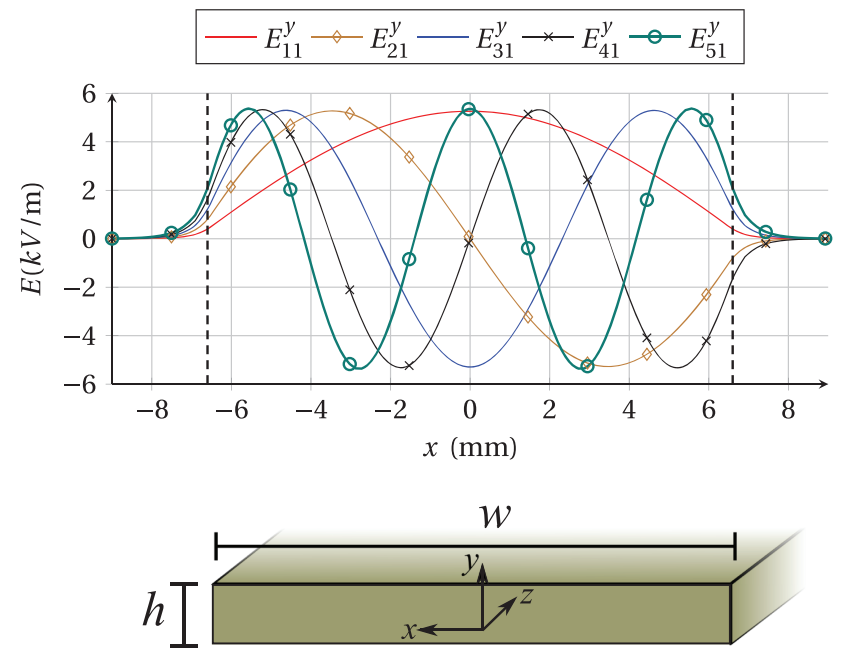

Figure 1: Mode profiles in $x$-direction of the first five modes in a multimode waveguide of width $w=12.25 \mathrm{~mm}$, height $h=1.8 \mathrm{~mm}$ and permittivity $\varepsilon_{r}=2.53$. The vertical dashed lines in the graph denote the waveguide's physical boundaries. The evanescent field components outside the dielectric are clearly visible.

to its large dimension of several wavelengths in width, i. e. $x$-direction, higher-order modes can be excited along $x$-direction, while the narrow height ensures no higherorder modes along $y$-direction.

It can be observed that the modes extend beyond the physical boundaries of the dielectric waveguide in a exponentially decaying manner due to the absence of metal, which would introduce a strict boundary. If more than one mode propagates in a waveguide, they start to superimpose constructively or destructively. Since all $\mathrm{m}$ propagating modes have different propagation constants $\beta_{m}$, the interference pattern shows spatial dependency in $z$-direction. Using paraxial approximations [14], the spacing of propagation constants between fundamental $(m=0)$ and $m$-th order mode is described by

$$
\beta_{0}-\beta_{m} \approx \frac{\pi m(m+2)}{3 L_{\pi}}
$$

where $L_{\pi}$ is the so called beat length of the multimode section [13]. It represents the coupling length of the two lowest-order modes $\beta_{0}$ and $\beta_{1}$ and can be determined by

$$
L_{\pi} \approx \frac{4 \sqrt{\varepsilon_{r}} w_{\mathrm{eff}}^{2},}{3 \lambda_{0}},
$$

where $\varepsilon_{r}$ denotes the relative permittivity of the dielectric, $w_{\text {eff }}$ represents the effective width of the multimode section and $\lambda_{0}$ is the free space wavelength. Note that the effective width of the multimode DWG has to be taken into 
account, since parts of the wave travel outside the dielectric (see Figure 1). With higher permittivity this width gets closer to the physical width of the multimode waveguide $w[13]$.

Based on the discussions in [13], the field distribution $\Psi(x, z)$ at length $z$ in the multimode section is represented by the summation of the field profile of all guided modes according to

$$
\Psi(x, z)=\sum_{m=0}^{M-1} c_{m} \psi_{m}(x) \exp \left[\frac{j m(m+2) \pi}{3 L_{\pi}} z\right],
$$

where $M$ denotes the maximum number of guided modes, $c_{m}$ and $\psi_{m}(x)$ describe the excitation coefficients and modal field distributions of the considered mode, respectively. Time dependency in the form of a factor $\exp (j \omega t)$ is implicitly assumed and not notated in the equation above. At $z=0$ the total field distribution is given by the input's field distribution. Therefore, at $z=0$ the sum of all propagable modes has to yield the input's field distribution. If the exponential in eq. (3) is equal to 1 or $(-1)^{m}$ the same (or mirrored) field distribution as at the feeding point is obtained, hence, a so-called self-image formed in the multimode waveguide. In general, these self-images occur at $z=L_{p}=p \cdot 3 L_{\pi}$ with $p=0,1,2 \ldots$. In between these distances higher-order images are formed. Without a particular feeding position the lengths for higher-order images are given in [13] as

$$
L_{p N}=\frac{p}{N} 3 L_{\pi},
$$

where $p \geq 0$ and $N \geq 1$ are integers with no common divisor. $N$ represents the number of images obtained at the corresponding length $L$. In general, these $N$ images have an amplitude of $\frac{1}{\sqrt{N}}$ with respect to the input field's amplitude and are not necessarily equally spaced throughout the width of the multimode waveguide. In order to have compact devices with shortest lengths, $p=1$ is assumed and will not be explicitly indexed throughout the rest of this paper. Based on the placement of the input waveguide along $x$, different lengths for self-imaging distances occur, since the input field's position restricts the excitation of certain modes. If the feed is placed directly in the center of the multimode section $(x=0)$, only $y$-axis-symmetric modes can be excited, which do not show a zero in the center. Hence only modes of odd order $m$ can be excited, i. e. $E_{11}^{y}, E_{31}^{y}$ and $E_{51}^{y}$ in Figure 1 .

Placing the feed in the center of the multimode waveguide leads to symmetric interference, described in [13]. The main benefit of this feed position is that the corresponding interference mechanism leads to shorter self-imaging lengths than any other feed position along $x$, which is why only central feeds are used for all devices introduced in this manuscript. Using symmetric interference, the length where $N$ images are formed is described by

$$
L_{N}=\left(3 L_{\pi}\right) / 4 N \text {, }
$$

which is a length four times smaller than in the general case. Figure 2 shows the interference pattern of a centrally fed MMI section, together with the distances $L_{1}=\frac{3}{4} L_{\pi}$, $L_{2}=\frac{3}{8} L_{\pi}$ and $L_{4}=\frac{3}{16} L_{\pi}$. If the device is cut at $z=L_{N}$ for power division, the maxima can arrive with different phases at the cutting plane. This is due to the differing physical distance from the central feed. In contrast to other interference-based effects, the MMI principle considered in this paper is relying on a single feed only and does not require a periodic structure, which is necessary, for example, for the Talbot effect [18].

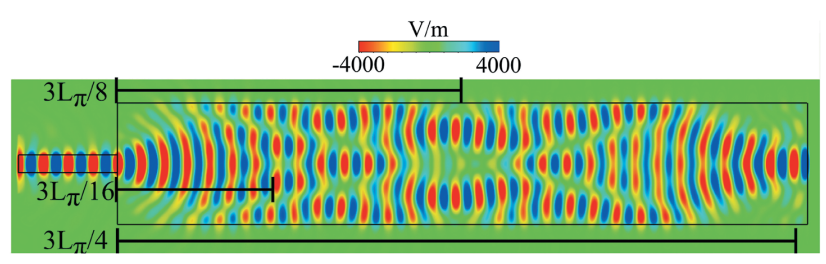

Figure 2: $E_{y}$ in a multimode waveguide of width $w=12.25 \mathrm{~mm}$ and permittivity $\varepsilon_{r}=2.53$ at $95 \mathrm{GHz}$. Different lengths for the occurrence of one, two and four maxima are indicated.

\section{One- and two-dimensional antenna arrays fed by integrated MMI power dividers}

Based on the discussions above, one- and twodimensional antenna arrays can be designed with integrated MMI power dividers. The antennas presented in this section are composed of Rexolite, a low-loss material of lower permittivity $\left(\varepsilon_{r, \operatorname{Rex}}=2.53, \tan \delta_{\operatorname{Rex}}=6 \cdot 10^{-4}\right.$ at $94 \mathrm{GHz}[19])$.

\section{$3.11 \times 4$ antenna array}

The first DRA array with integrated power split achieved by MMI was proposed in [15] and forms a $1 \times 4$ array. Its dimensions are given in Figure 3.

The feed is realized as a subwavelength DWG with edge length $a=1.8 \mathrm{~mm}$ and is located at the center of 


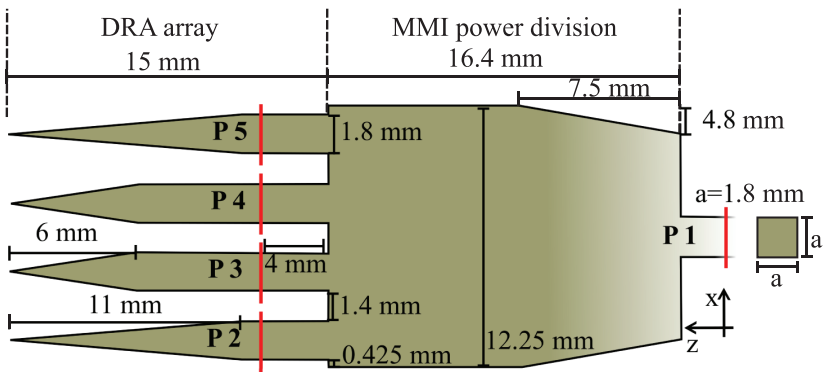

Figure 3: Schematic with corresponding dimensions of the $1 \times 4$ DRA array with integrated power divider.

the power divider section. Its dimensions are found by Marcatili's approximation method [20] and are adjusted with CST Studio Suite, such that the subwavelength DWG big enough for mechanical handling and stability, but mono-mode propagation is still maintained. In order to mitigate radiation losses, the MMI section is not instantly widened to its maximum width $w=12.25 \mathrm{~mm}$ but has a short tapered section. Note, that an abrupt change in width is essential for MMI to be triggered, hence, the taper shall not reach the subwavelength DWG. The necessary length for the formation of four images is calculated according to eq. (5) and fine-tuned by full-wave simulations performed with CST Studio Suite. Since the formation of maxima is due to interference, the integrated power divider is inherently band limited. The length $L_{4}$ necessary for four maxima of equal intensity is dependent on $L_{\pi}$, which is in turn depending on the effective wavelength $\lambda_{\text {eff }}=\lambda_{0} / \sqrt{\varepsilon_{r}}$ according to eq. (2). Therefore, $L_{4}$ is a frequency dependent variable. This circumstance manifests in varying S-parameters for each port, visualized in Figure 4. It strikes that the outer ports P2 and P5 share similar behavior, as well as the inner ports $\mathrm{P} 3$ and $\mathrm{P} 4$ do. This behavior is present in phase as well. Power splits with an imbalance of less than $1 \mathrm{~dB}$ are achieved in a range from $90 \mathrm{GHz}$ to $105 \mathrm{GHz}$.

With the power divider at hand, the antenna elements have to be investigated. Most important is to compensate the phase difference of the output ports introduced by MMI according to Figure 2. In order to do so, the DRA elements have a difference in taper length. The DRAs at the outer ports, behind in phase, have a longer taper length than the DRAs at inner ports. Therefore, the propagation constant of a wave traveling on the DRA with longer taper is earlier adopted to the one of free space. Choosing the taper lengths carefully, a nearly plane phase front can be achieved at the tips of the DRAs. This behavior is visualized in Figure 5. The antenna elements were chosen to have a spacing of $3.2 \mathrm{~mm}\left(1.01 \lambda_{0}\right.$ at $\left.95 \mathrm{GHz}\right)$ in order to reduce coupling.

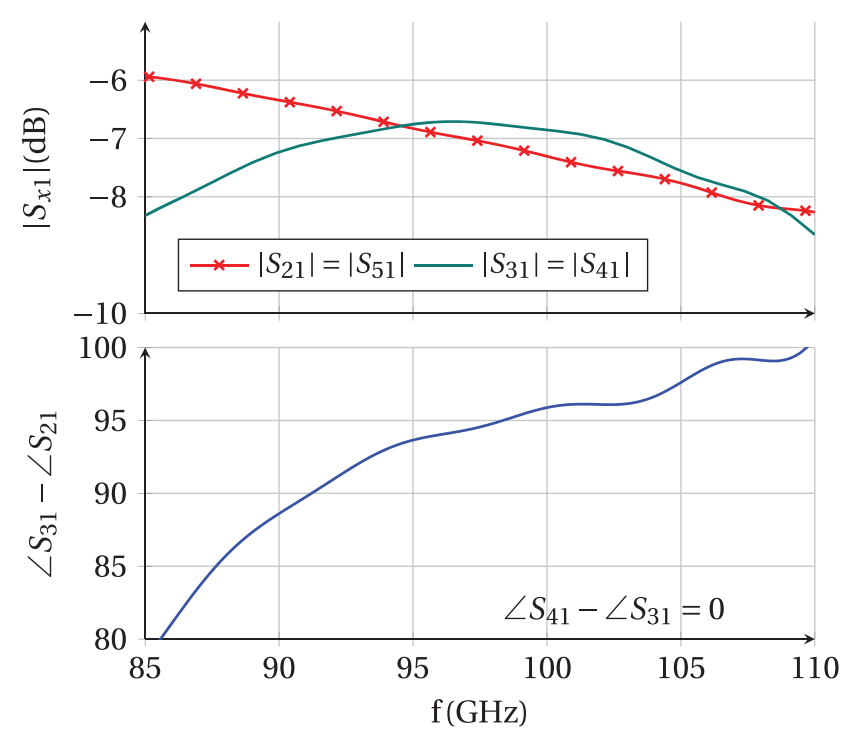

Figure 4: Simulated S-parameters in magnitude (top) and their corresponding phase difference (bottom). Pairwise similar behavior of the output ports can be observed. The central ports (P3 and P4) are ahead in phase with respect to the outer ports (P2 and P5) [15].

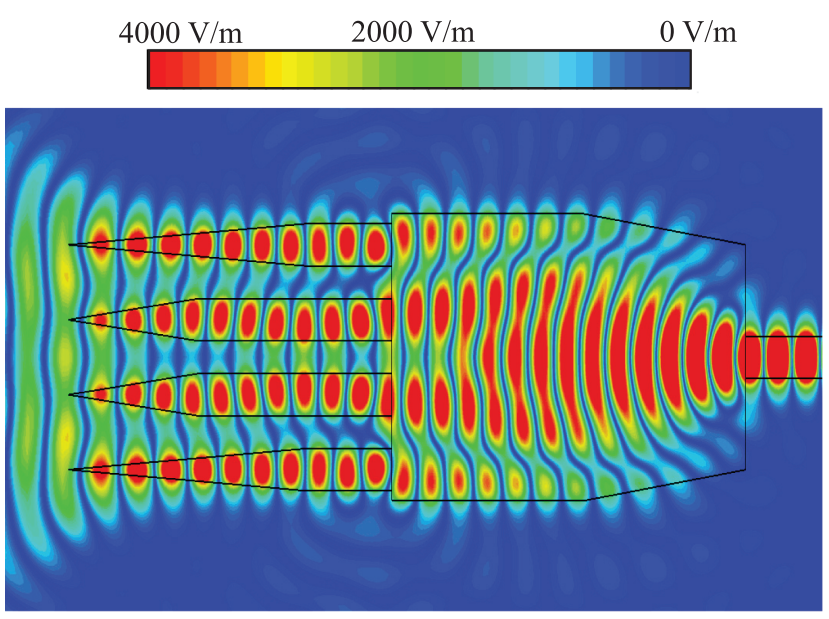

Figure 5: Simulated absolute value of the E-field along the fully dielectric DRA array at $95 \mathrm{GHz}$.

Figure 6a shows the fabricated antenna, milled from Rexolite in one piece. For measurements the dielectric feed is connected to a WR10 hollow-waveguide-to-dielectricwaveguide transition [21], [22]. The antenna together with the waveguide transition is mounted on a rotary table for pattern characterization (Figure 6b). The reference antenna is a $22 \mathrm{dBi}$ standard gain horn antenna.

The measured, individually normalized antenna pattern for selected frequencies are given in Figure 7. Good agreement between simulation and measurement is achieved. In E-plane, the pattern is formed by the chosen rod shape, while in H-plane the rod array determines the 


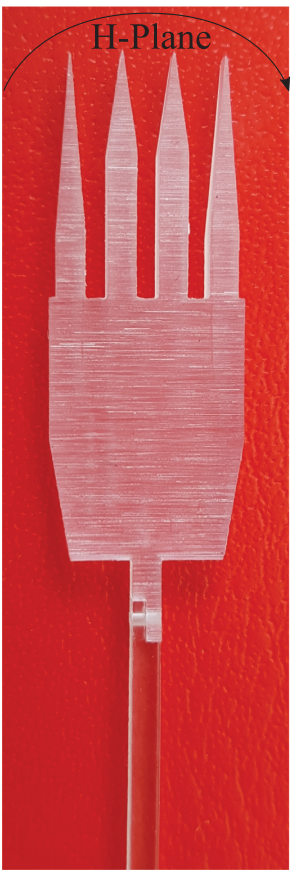

(a)

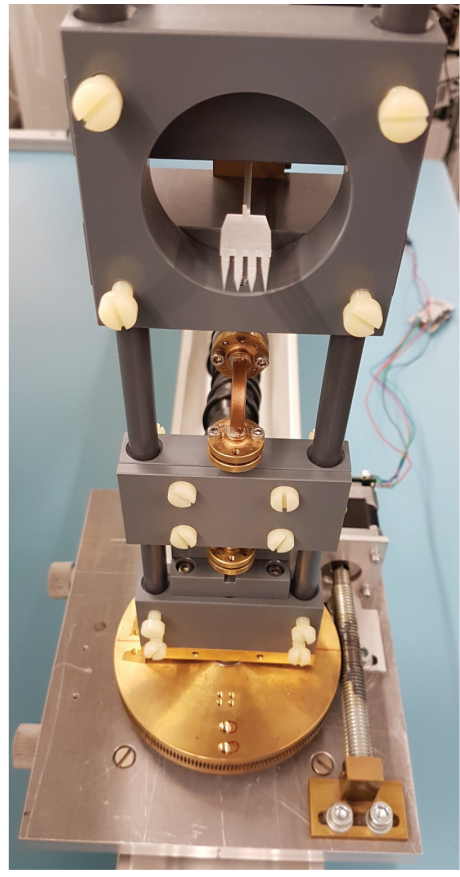

(b)
Figure 6: (a) DRA array milled from Rexolite which is (b) mounted on the rotary table for pattern characterization [15].

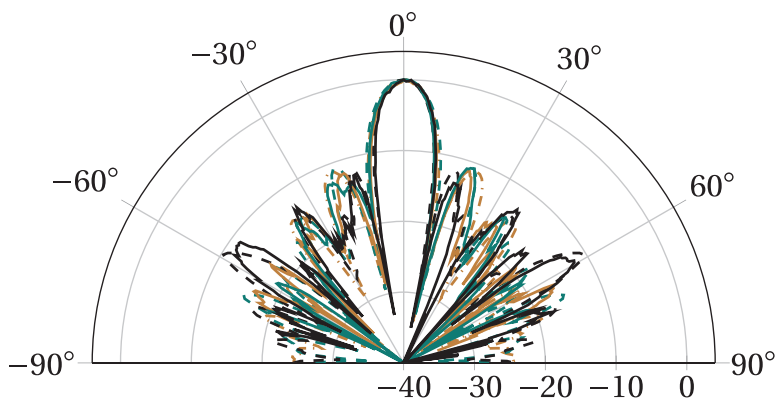

e
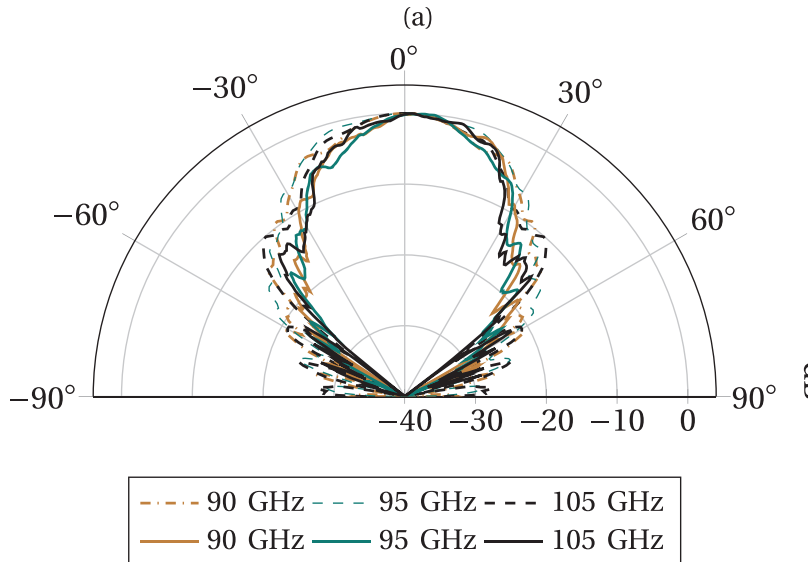

(b)

Figure 7: Individually normalized measured pattern of the $1 \times 4$ DRA array at selected frequencies, compared with simulation results (dashed). (a) H-plane shows narrow beam width due to the array while (b) E-plane is dominated by the pattern of a single element [15].

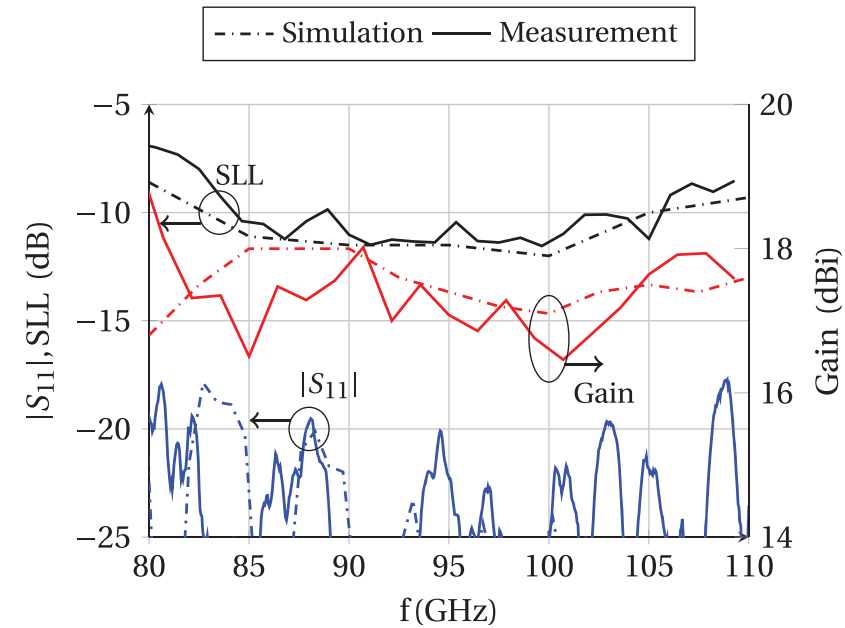

Figure 8: Gain, SLL and input reflection of the $1 \times 4$ DRA array over frequency [15].

pattern. An overview of the frequency dependent behavior regarding gain, sidelobe level (SLL) and input reflection is given in Figure 8. The measured gain is between $16.5 \mathrm{dBi}$ and $18 \mathrm{dBi}$, while the SLL stays below $-10 \mathrm{~dB}$ in the design range.

\section{$3.24 \times 4$ antenna array}

The concept of MMI can be extended to a two-dimensional approach. As described in [23], the field expression in eq. (3) is extended to represent higher-order mode propagation in the second dimension, hence forming:

$$
\begin{aligned}
& \Psi(x, y, z)= \\
& \sum_{k=0}^{K-1} \sum_{m=0}^{M-1} c_{k m} \psi_{k m}(y) \exp \left[\frac{j m(m+2) \pi z}{3 L_{x \pi}}\right] \exp \left[\frac{j k(k+2) \pi z}{3 L_{y \pi}}\right] .
\end{aligned}
$$

$m$ and $k$ denote the order of the two dimensional modes $\beta_{m k}$ for $x$ - and $y$-direction, respectively. The two exponentials describe dependency on the beat length and mode order in $x$ - and $y$-direction. For an axis-symmetric device $L_{x \pi}=L_{y \pi}=L_{\pi}$, since the effective widths of the MMI section are the same in both directions $\left(w_{\text {eff } x}=w_{\text {eff } y}\right)$. Therefore, for central feed positioning, one particular length for the formation of $N$ maxima in both $x$ - and $y$-direction can be calculated: $L_{N N}=\left(3 L_{\pi x}\right) / 4 N_{x}=\left(3 L_{\pi y}\right) / 4 N_{y}=\left(3 L_{\pi y}\right) / 4 N$. Figure 9 depicts the model of the two-dimensional power divider with 16 output ports, proposed in [16], and its corresponding dimensions. Due to the symmetric design, it is sufficient to investigate four out of 16 output ports. Similar to the one-dimensional case, even if the ports 

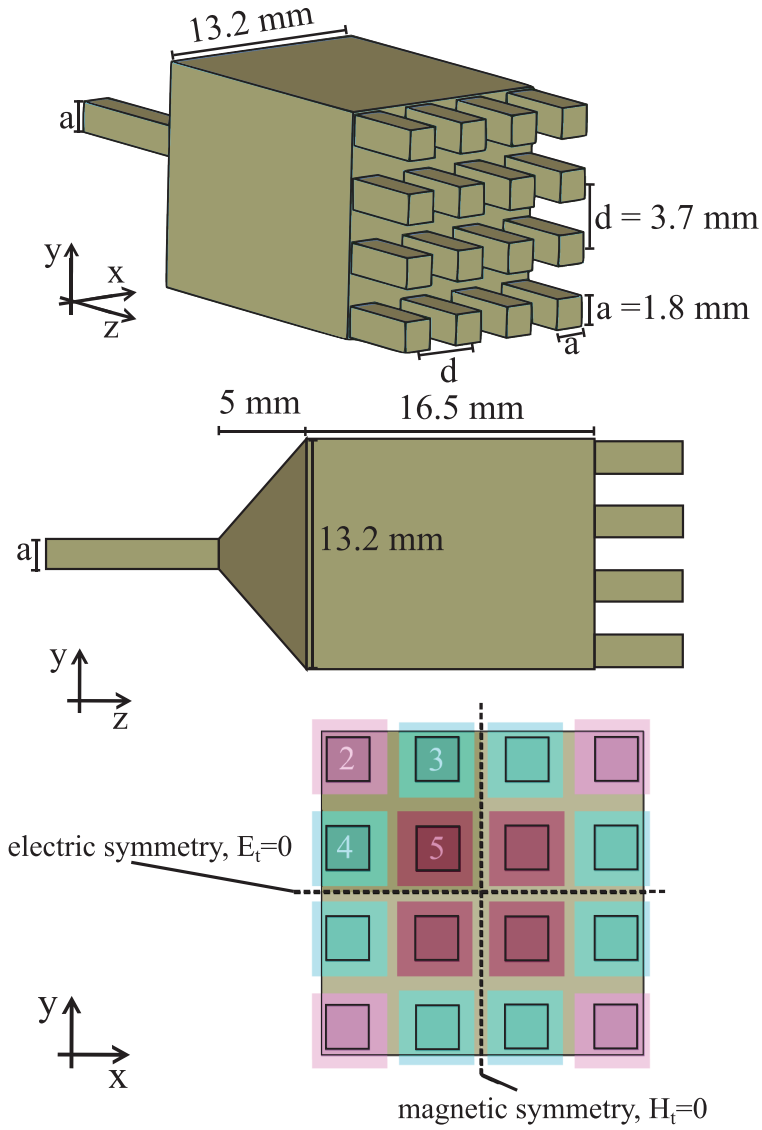

Simulated phase relations with respect to Port 5 at $92.5 \mathrm{GHz}$ :
$\varphi=0^{\circ}$
$\varphi=+90^{\circ}$
$\varphi=+180^{\circ}$

Figure 9: Schematic of the two-dimensional power divider with its corresponding dimensions. The 16 output ports, showing equal output power, can be classified into three groups, depending on the corresponding phase (see color code).

are well balanced, their phase differs, depending on the port's distance to the center of the MMI section. Inner ports are ahead in phase compared to ports having a larger radial distance from the central point of the divider.

In order to verify a $1 / 16$ power split in practice and the corresponding phase distributions as simulated, the $1: 16$ power divider was milled in one piece from Rexolite. Since only two ports could be simultaneously measured in the lab, while all other ports have to be well matched, adaptable port access has to be accomplished. This is done by using a pin-plug structure in order to be able to connect the measurement probe or a radiation element to the ports. If a port is measured, a dielectric output is connected while all other ports are terminated with a radiation taper, radiating away from the measured port. This measurement principle is depicted in Figure 10. A

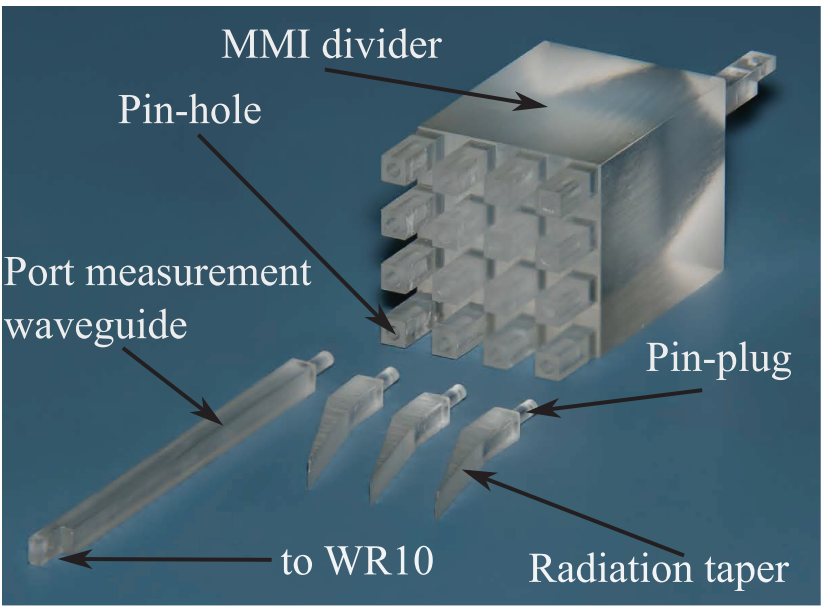

(a)

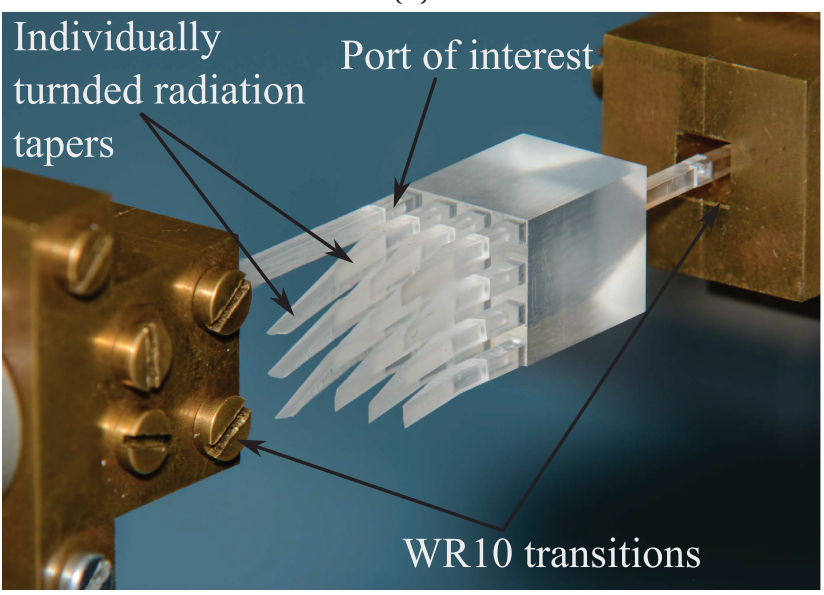

(b)

Figure 10: (a) Fabricated parts for characterization and (b) characterization setup of the 2D MMI power divider. The radiation tapers can be turned such that they will always radiate away from the WR10 transition, so the measurement is not disturbed.

more detailed description of the measurement is provided in [16]. Measurement results regarding magnitude and phase of the output ports are given in Figure 11. High agreement is achieved for the phase distribution, while agreement differs in magnitude at the upper frequency band. Deviations may be caused by small misalignments when measuring the different ports. All 16 ports are measured and the symmetrical behavior could be verified. The input reflection is below $-15 \mathrm{~dB}$ up to $105 \mathrm{GHz}$. In order to use the divider for an antenna array, three different phase offsets have to be compensated in the $4 \times 4$ array. Again, we make use of different taper lengths of the DRAs in to achieve phase alignment. Therefore, with the four inner ports (red marked in Figure 9) forming the reference phase and being connected to DRAs with a certain taper length, the ports $90^{\circ}$ behind (cyan in Figure 9) 


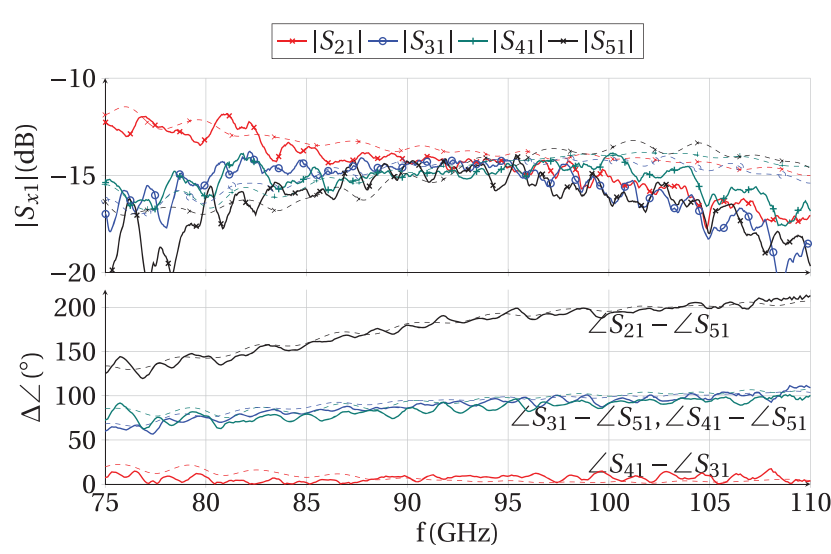

Figure 11: Output power levels and corresponding phases for the ports defined in Figure 9. Simulated results are depicted with dashed lines [16].

have to be connected to DRAs with longer taper length and the ports at the four edges of the array have to be connected to DRAs with the longest taper length. These lengths have been optimized with the help of CST Studio Suite. The detailed rod lengths can be found in [16]. The DRAs show the same plug structure used for measuring the single ports and can be easily connected to the fully characterized power divider. The two-dimensional power divider shows an element spacing of $3.7 \mathrm{~mm}\left(\approx 1.17 \lambda_{0}\right.$ at $95 \mathrm{GHz}$ ) for decoupled output ports. This distance is given by the milling process when the device is fabricated in one piece. The assembled array is depicted in Figure 12.

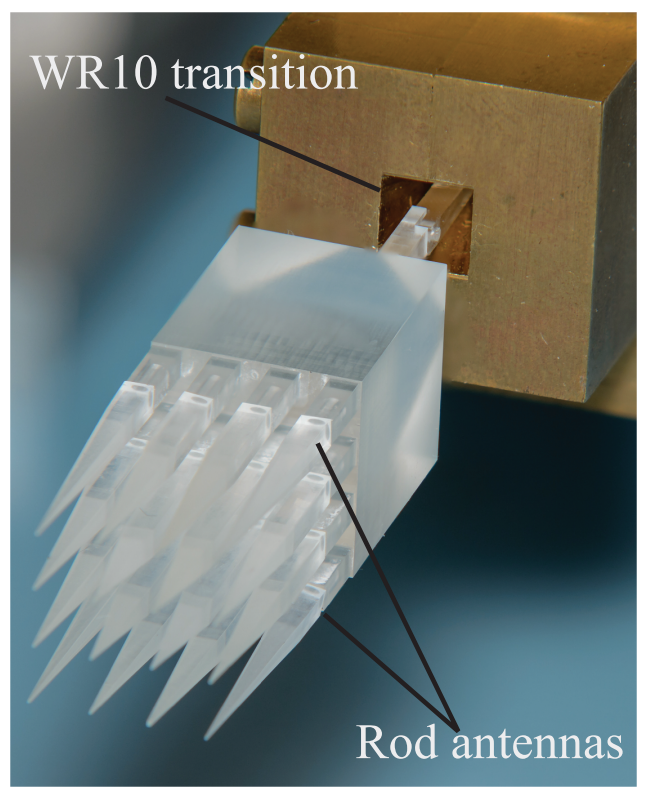

Figure 12: Fully assembled $4 \times 4$ DRA array with integrated 2D power divider, connected to a WR10 transition. This setup is mounted on a rotary table for pattern acquisition [16].

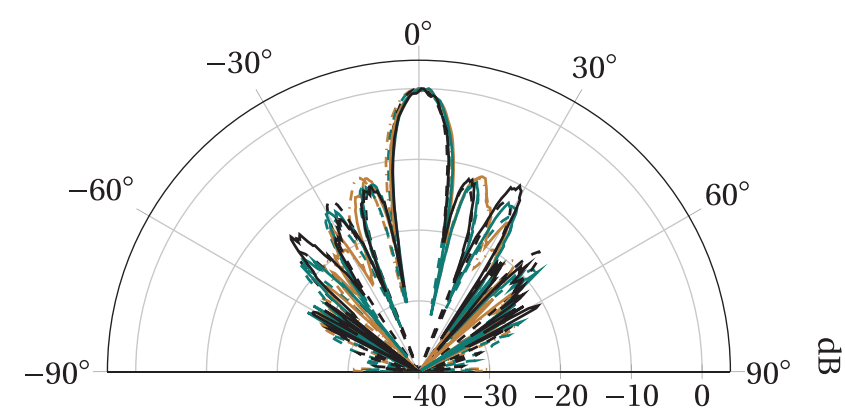

(a)
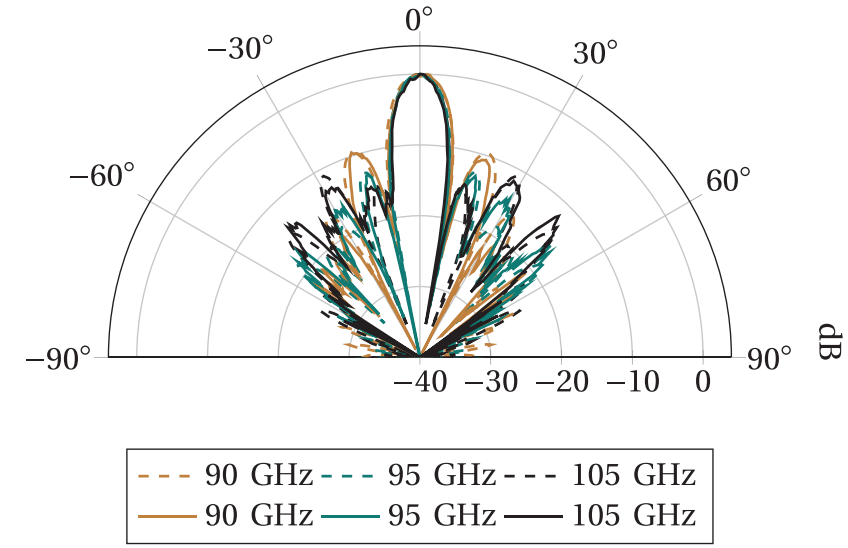

(b)

Figure 13: Individually normalized measured pattern of the $4 \times 4$ DRA array at selected frequencies, compared with simulation results (dashed). (a) H-plane and (b) E-plane show similar behavior [16].

Figure 13 depicts the antenna array together with the WR10 transition necessary for measurements. The pattern is measured in the same way as the $1 \times 4$ DRA array's pattern. Figure 13 shows the measured, individually normalized pattern in E- and H-plane. In contrast to the $1 \times 4 \mathrm{DRA}$ array, the E-plane pattern is now narrowed as well, due to the second dimension of the array. The behavior of gain, SLL in H-plane and input reflection is given in Figure 14. Compared with the results of the $1 \times 4$ array (Figure 8 ) similar SLL behavior and up to $4.5 \mathrm{~dB}$ higher gain at $97.5 \mathrm{GHz}$ are achieved. An increased input reflection is observed, however, it is still below $-15 \mathrm{~dB}$. Gain is approximately $2 \mathrm{~dB}$ lower than simulated. This can be caused by the amount of individual DRAs with fabrication tolerances connected to the 16 output ports. The reconfigurable port access (pin-plug structure) can introduce slight losses, and phase may not be exactly compensated. These influences can be eliminated if array and power divider are manufactured from one piece, as Figure 8 indicates, where simulated and measured gain show less deviations. Especially 3D printing or injection molding can prove advantageous when considering the production of a two-dimensional structure. 


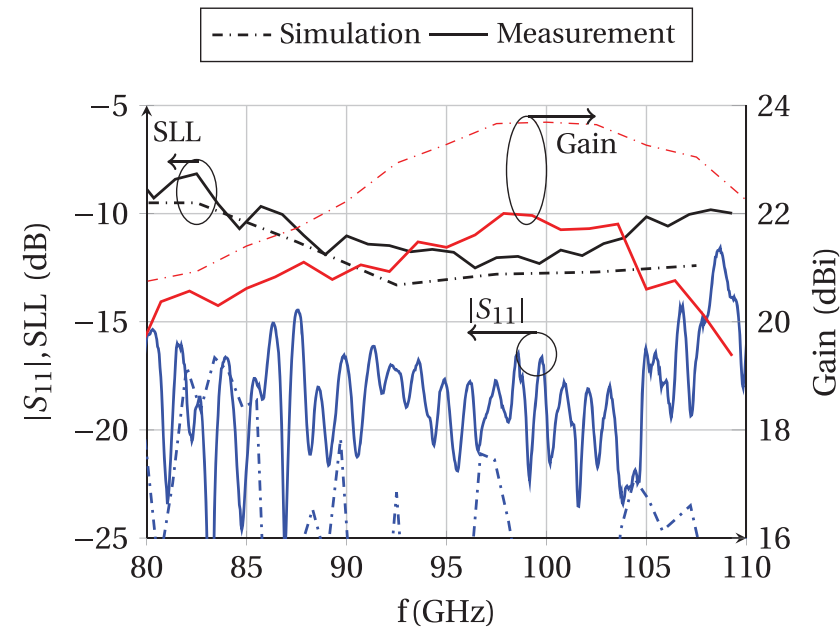

Figure 14: Gain, SLL and input reflection of the $4 \times 4$ DRA array over frequency.

\section{Feasibility analysis of $1 \times 4$ antenna arrays with materials of higher permittivity}

It has been shown in [24] that a controlled phase difference along the DRA elements provides reasonable beam steering capabilities. A phased array can provide reconfigurable phase relations to steer the beam in different directions, but grating lobes have to be suppressed by moving the antenna elements closer together. In order to assess miniaturization and beamsteering capabilities, higher permittivity materials are investigated. For simplicity reasons regarding design, fabrication and verification, only one-dimensional antenna arrays are considered. They are then compared to the $1 \times 4$ array introduced in the previous Section.

The design is straightforward according to the theory described in Section 2 and Section 3. Detailed information can be found in [17]. Since the permittivity increases, the dimensions of the single mode fiber decrease. Similarly, the overall dimensions of the MMI section decrease, allowing more narrow placement of the output ports, and therefore, the antenna elements. The materials considered are Premix' Preperm L300 and L440, whose successors are now known as Preperm PPE300 and PPE440 [25], respectively, showing a relative permittivity of $\varepsilon_{r, \mathrm{~L} 300}=3.0$ and $\varepsilon_{r, \mathrm{~L} 440}=4.4$. Important parameters of the materials and the antenna arrays are compared in Table 1. All arrays are realized as one piece, milled from the corresponding materials, and are depicted in Figure 15. A reduction of the sectional area of 52\% is achieved when using Preperm L440 instead of Rexolite. Correspondingly, the weight of
Table 1: Comparison of DRA arrays composed of Rexolite, Preperm L300 and Preperm L440. For definition of variables see Figure 15.

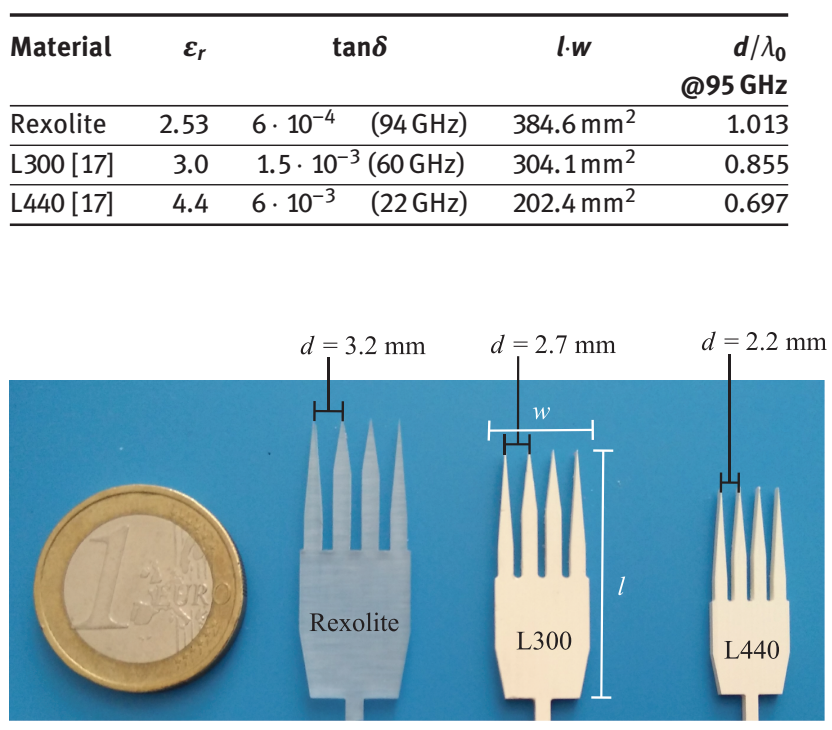

Figure 15: DRA arrays made from three different materials.

the array $(0.5 \mathrm{~g})$ is reduced by $30 \%$ to $0.35 \mathrm{~g}$. In addition, closer element spacing $d$ of $0.697 \lambda_{0}$ at $95 \mathrm{GHz}$ instead of $1.013 \lambda_{0}$ can be realized. All three arrays are well matched according to Figure 16. In fact, especially in the case of L440, simulations predicted worse matching than measured, highlighting the uncertainty of the loss tangent of the material in the measurement range. In addition, both high permittivity structures had to be manually adjusted to fit to the WR10 transition, which increases tolerances.

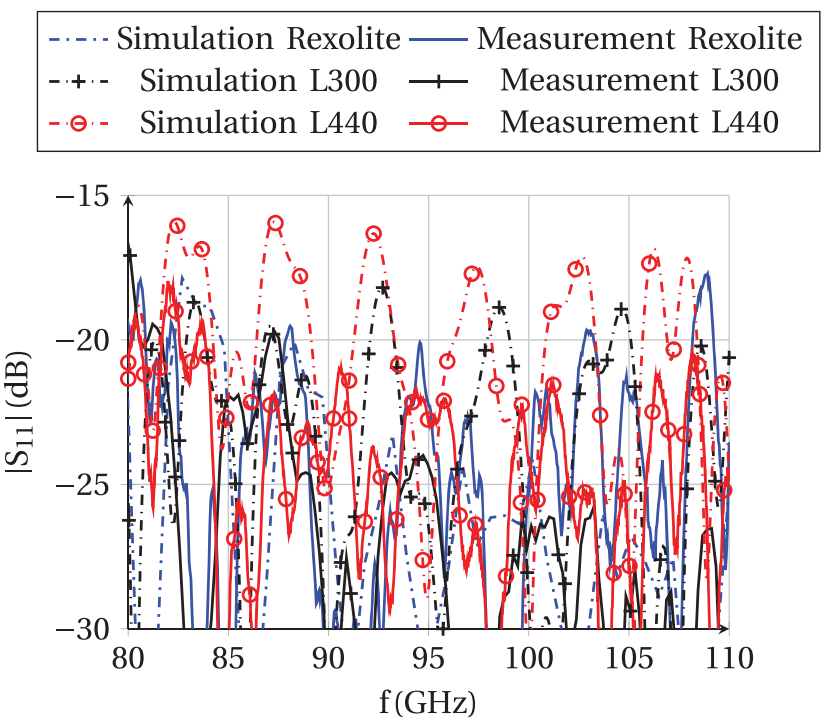

Figure 16: Simulated and measured input reflection of three $1 \times 4$ DRA arrays made from different materials. 


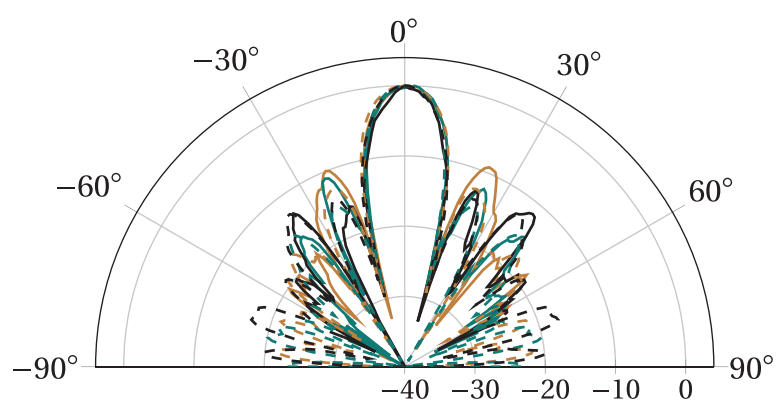

(a)

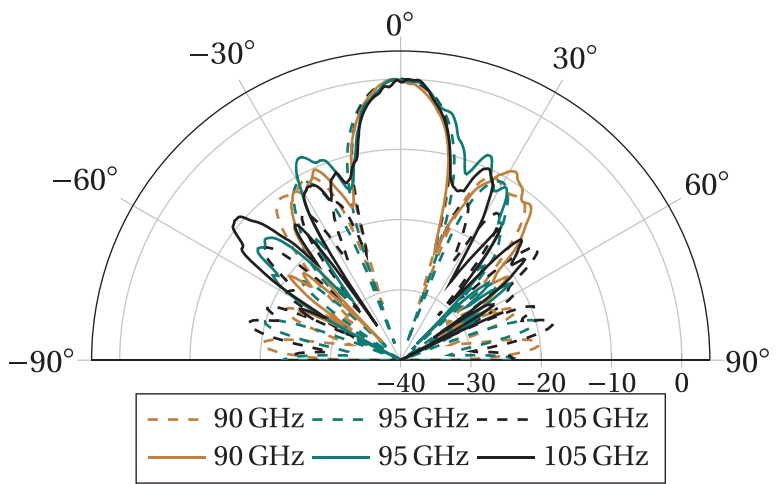

(b)

Figure 17: Individually normalized $\mathrm{H}$-plane pattern of $1 \times 4$ DRA arrays composed of (a) Preperm L300 and (b) Preperm L440 at selected frequencies, compared with simulation results (dashed) [17].

\begin{tabular}{|lccc}
\hline$-*-$ & SLL, L300 & $*$ & Gain, L300 \\
$-\odot-$ & SLL, L440 & - & Gain, L440 \\
--- & SLL, Rexolite & & Gain, Rexolite
\end{tabular}

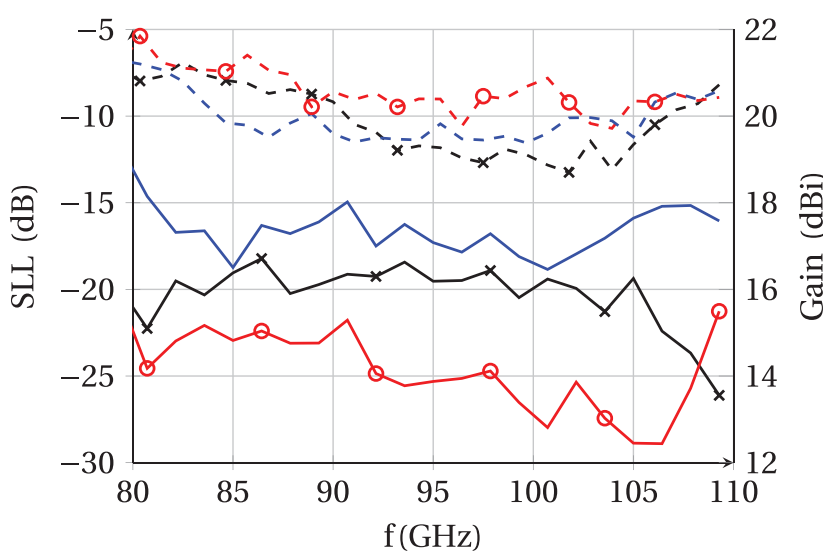

Figure 18: Measured gain and SLL for three $1 \times 4$ DRA arrays made from different materials.

The pattern obtained in H-Plane for both Premix materials are given in Figure 17. They are measured as described in Section 3. Figure 18 shows a comparison of measured gain and SLL for all three materials, indicating decreasing gain with increasing permittivity. This is due to the more lossy high permittivity materials. In addition, since the evanescent components decrease with higher permittivity, the wave is more concentrated in the dielectric with increasing permittivity. Therefore, higher losses have even more impact. In general, due to the smaller aperture size of the antennas when decreasing their physical size, the main beam broadens (c.f. Figure 17 and Figure 7a), and gain decreases. SLLs stay in the same order with all materials. L440 is inferior to the two other materials regarding gain and SLL throughout the whole frequency range. This can be due to the fact that the losses around $100 \mathrm{GHz}$ of L300/L440 were unknown at the time of measuring [17]. The now available successor materials, PPE300 and PPE440 [25], are characterized up to $60 \mathrm{GHz}$ and show losses reduced by a factor of 2 in the case of PPE440 compared to L440. Tolerances of approximately $50 \mu \mathrm{m}$ during the milling process have higher impact in the high permittivity designs since overall dimensions are smaller. In addition, the WR10 transition is not fully optimized for a permittivity of $\varepsilon_{r, \text { PPE } 440}$. Nevertheless, it could be shown that increasing permittivity can be used in order to produce compact DRA arrays, also suitable for phased array applications. For an MMI fed antenna array with an element spacing $d \leq 0.5 \lambda_{0}$ at $95 \mathrm{GHz}$ a material of $\varepsilon_{r} \approx 6$, such as Premix' PPE650 [25] $\left(\varepsilon_{r}=6.5\right.$ at $\left.60 \mathrm{GHz}\right)$ can be used. In order to realize a phased array, phase shifters could be introduced in the DRA elements by using microwave liquid crystal, which has already been successfully combined with dielectric components [26].

\section{Conclusion}

This paper presents an overview of fully dielectric antenna arrays with integrated power dividers. The key mechanism for these compact devices is multi-mode interference (MMI), which allows both one- and two-dimensional, equal power splits in a single step. Using specific lengths of the multimode waveguide, different amounts of equally spaced maxima of equal power can be formed and distributed to single-mode output waveguides. A possible application is to utilize the MMI power divider as a feeding structure for antenna arrays, e. g. made of dielectric rod antennas (DRAs). DRAs are demonstrated to be an easy to control antenna element, since the single mode waveguides have to be tapered only in order to form an antenna. As power splits are of equal magnitude but not necessarily of equal phase, phase adjustment is mandatory for a satisfying antenna pattern and gain. 
Therefore, the DRAs show different taper lengths. Oneand two-dimensional DRA arrays are proposed in this paper, with gain, ranging between $12.5 \mathrm{dBi}$ and $22 \mathrm{dBi}$, depending on array size, frequency and material. In the design range of $90 \mathrm{GHz}$ to $105 \mathrm{GHz}$ the sidelobe levels are below $-10 \mathrm{~dB}$, with the exception of the array made from Preperm L440. The proposed materials of higher permittivity, L300 and L440, show a reduction of size and weight, as well as a reduction of element spacing, while the overall performance could be kept similar to the lower permittivity $1 \times 4$ Rexolite array. Throughout all proposed designs the simulations show good agreement with the measurements, indicating reliable design possibilities.

In the case of the two-dimensional array, a measurement setup for power divider characterization has been introduced by the proposed pin-plug structure, which allows interchangeable connections to the outputs of the power divider. High gain of $22 \mathrm{dBi}$ could be achieved.

The necessary multimode waveguide can be easily fabricated from plastics in one piece. In this work, fabrication is realized by CNC-milling, but other concepts such as injection molding or 3D printing are possible alternatives well suited for mass production. At lower frequencies (up to $11 \mathrm{GHz}$ ) several 3D printed materials have already been characterized [27]. In [28], a 3D printed Alumina structure operating at $\mathrm{W}$-band is presented and compared to counterparts of different materials created by classical manufacturing techniques.

Acknowledgment: The authors would like to thank CST AG, Germany, for providing CST Studio Suite and Premix Oy, Rajamäki, Finland, for supply of the Preperm materials.

The authors are with the Institute for Microwave Engineering and Photonics, Technische Universitaet Darmstadt, Germany.

\section{References}

[1] M. Geiger, M. Hitzler, S. Saulig, J. Iberle, P. Hugler and C. Waldschmidt, "A 160-GHz radar with flexible antenna used as a sniffer probe," IEEE Sens. J., vol. 17, no. 16, pp. 5104-5111, Aug. 2017.

[2] H.-U. Nickel and J. Zovo, "Novel flexible dielectric waveguide for millimeter and sub-millimeter frequencies - design and characterization," in 84th ARFTG Microwave Meas. Conf., IEEE, Dec. 2014.

[3] M. Jost, R. Reese, M. Nickel, H. Maune and R. Jakoby, "Fully dielectric interference-based SPDT with liquid crystal phase shifters," IET Microwaves Antennas Propag., vol. 12, no. 6, pp. 850-857, May 2018.
[4] R. Reese, M. Jost, H. Maune and R. Jakoby, "Design of a continuously tunable w-band phase shifter in dielectric waveguide topology," in 2017 IEEE MTT-S Int. Microwave Symp. (IMS), IEEE, Jun. 2017.

[5] D. Chicherin, M. Sterner, J. Oberhammer, S. Dudorov, D. Lioubtchenko, A. J. Niskanen, V. Ovchinnikov and A. V. Räisänen, "MEMS based high-impedance surface for millimetre wave dielectric rod waveguide phase shifter," in Proc. 40th Eur. Microwave Conf., Sep. 2010, pp. 950-953.

[6] A. A. Generalov, D. V. Lioubtchenko and A. V. Räisänen, "Reconfigurable $\mathrm{mm}$-wave phase shifter based on high impedance surface with carbon nanotube membrane MEMS," in Proc. Global Symp. Millimeter-Waves (GSMM), May 2015, pp. 1-3.

[7] S. Kobayashi, R. Mittra and R. Lampe, "Dielectric tapered rod antennas for millimeter-wave applications," IEEE Trans. Antennas Propag., vol. 30, no. 1, pp. 54-58, Jan. 1982.

[8] J. P. Pousi, D. V. Lioubtchenko, S. N. Dudorov and A. V. Raisanen, "High permittivity dielectric rod waveguide as an antenna array element for millimeter waves," IEEE Trans. Antennas Propag., vol. 58, no. 3, pp. 714-719, Mar. 2010.

[9] A. Rivera-Lavado, S. Preu, L. E. Garcia-Munoz, A. Generalov, J. M. de Paz, G. Dohler, D. Lioubtchenko, M. Mendez-Aller, S. Malzer, D. Segovia-Vargas and A. V. Raisanen, "Array of dielectric rod waveguide antennas for millimeter-wave power generation," in 2015 Eur. Microwave Conf. (EuMC), IEEE, Sep. 2015.

[10] Y. Konishi, Y. Aramaki, S. Yamaguchi, I. Naito, N. Yoneda and M. Ohtsuka, "Millimeter-wave waveguide-type array antennas using low-loss engineering plastics," in Proc. 3rd Eur. Conf. Antennas Propag., Mar. 2009, pp. 520-524.

[11] M. Jost, R. Reese, J. Pauls, J. S. K. Gautam, R. Gemble, C. Weickhmann, O. H. Karabey and R. Jakoby, "Comparison of hollow waveguide and dielectric fibre based spdt switches for w-band," in Proc. German Microwave Conf. (GeMiC), Mar. 2016, pp. 140-143.

[12] S. Raghuwanshi, V. Kumar, D. Chack and S. Kumar, "Propagation study of y-junction optical splitter using BPM," in 2012 Int. Conf. Commun. Sys. Network Technol., IEEE, May 2012.

[13] L. B. Soldano and E. C. M. Pennings, "Optical multi-mode interference devices based on self-imaging: principles and applications," J. Lightwave Technol., vol. 13, no. 4, pp. 615-627, Apr. 1995.

[14] M. Bachmann, P. A. Besse and H. Melchior, "General self-imaging properties in $\mathrm{n} \times \mathrm{n}$ multimode interference couplers including phase relations," Appl. opt., vol. 33, no. 18, pp. 3905-3911, 1994.

[15] R. Reese, M. Jost, M. Nickel, E. Polat, R. Jakoby and H. Maune, "A fully dielectric lightweight antenna array using a multimode interference power divider at w-band," IEEE Antennas Wirel. Propag. Lett., vol. 16, pp. 3236-3239, 2017.

[16] R. Reese, H. Tesmer, M. Jost, E. Polat, M. Nickel, R. Jakoby and H. Maune, "A compact two-dimensional power divider for a dielectric rod antenna array based on multimode interference," J. Infrared Millimeter Terahertz Waves, vol. 39, no. 12, pp. 1185-1202, Aug. 2018.

[17] R. Reese, H. Tesmer, E. Polat, M. Jost, M. Nickel, R. Jakoby and H. Maune, "Fully dielectric rod antenna arrays with high 
permittivity materials," in 2019 12th Ger. Microwave Conf. (GeMiC), IEEE, Mar. 2019.

[18] H. Talbot, "LXXVI. facts relating to optical science. no. IV," London Edinburgh Dublin Philos. Mag. J. Sci., vol. 9, no. 56, pp. 401-407, Dec. 1836.

[19] G. Friedsam and E. Biebl, "Precision free-space measurements of complex permittivity of polymers in the w-band," in 1997 IEEE MTT-S Int. Microwave Symp. Dig., IEEE.

[20] E. A. J. Marcatili, "Dielectric rectangular waveguide and directional coupler for integrated optics," Bell Syst. Tech. J., vol. 48, no. 7, pp. 2071-2102, Sep. 1969.

[21] C. Yeh and F. I. Shimabukuro, The Essence of Dielectric Waveguides. Springer US, 2008, pp. 85-87.

[22] T. Trinh, J. Malherbe, and R. Mittra, “A metal-to-dielectric waveguide transition with application to millimeter-wave integrated circuits," in 1980 IEEE MTT-S International Microwave symposium Digest. IEEE, 1980, pp. 205-207.

[23] D. Khalil and A. Yehia, "Two-dimensional multimode interference in integrated optical structures," J. Opt. A: Pure Appl. Opt., vol. 6, no. 1, pp. 137-145, Nov. 2003. [Online].
Available: https://doi.org/10.1088\%2F1464-4258\%2F6\% 2F1\%2F025

[24] R. Reese, M. Jost, E. Polat, M. Nickel, R. Jakoby and H. Maune, "Beam steering capabilities of a fully dielectric antenna array," in Proc. IEEE Int. Symp. Antennas Propag. USNC/URSI National Radio Sci. Meeting, Jul. 2018, pp. 2187-2188.

[25] Premix Oy, Finland. [Online]. Available: https://www.preperm .com/products/raw-materials/

[26] H. Maune, M. Jost, R. Reese, E. Polat, M. Nickel and R. Jakoby, “Microwave liquid crystal technology," Crystals, vol. 8(9), no. 355, Sep. 2018.

[27] P. I. Deffenbaugh, R. C. Rumpf and K. H. Church, "Broadband microwave frequency characterization of 3-d printed materials," IEEE Trans. Compon. Packag. Manuf. Technol., vol. 3, no. 12, pp. 2147-2155, Dec. 2013.

[28] A. Jimenez-Saez, M. Schubler, C. Krause, D. Pandel, K. Rezer, G. V. Bogel, N. Benson and R. Jakoby, "3D printed alumina for low-loss millimeter wave components," IEEE Access, vol. 7, pp. 40 719-40724, 2019. 